\section{Neu im Amt}

Rainer Manske ist neuer Pflegedirektor im BG Klinikum Unfallkrankenhaus Berlin $\mathrm{gGmbH}$, einem hoch spezialisierten klinischen Zentrum zur Behandlung Schwerkranker und zur Rettung und Rehabilitation Schwerverletzter aus dem gesamten Bundesgebiet.

\section{? Herr Manske, Sie sind seit dem 1. Juli} 2016 Pflegedirektor im Unfallkrankenhaus Berlin. Was hat Sie motiviert, diese Stelle anzunehmen?

Manske: Ich war schon zu einem früheren Zeitpunkt meiner beruflichen Laufbahn als Pflegeleitung des Notfallzentrums im Unfallkrankenhaus Berlin (ukb) tätig. Daher hatte ich eine ungefähre Vorstellung von dem, was auf mich zukommen wird. Im Anschluss daran habe ich zehn Jahre als Pflegedirektor in Kliniken der Paracelsius und Helios Gruppe in Norddeutschland gearbeitet. Durch diese Leitungsfunktionen habe ich weiteres Wissen erworben und neue Erfahrungen gemacht, mit allen dazugehörigen Kompetenzen.

? Was zeichnet aus Ihrer Sicht das Pflegefachpersonal des ukb aus?

Manske: Wir verfügen über ein gut ausgebildetes und hoch spezialisiertes Personal. Das ist unbedingt erforderlich, weil Wissen eines unserer zentralen Themen ist. Ein Versorgungsanbieter wie das ukb zeichnet sich als Wissenskonzern aus. Aktuelles Wissen, das gekonnt praktisch umgesetzt wird, sichert den nötigen Wettbewerbsvorteil am Gesundheitsmarkt. Darum sind wir an einer guten, wissenschaftlich fundierten Grundausbildung und einer kontinuierlichen Fort- und Weiterbildung in der Pflege interessiert. Dafür will sich das ukb mit seinen Ausbildungspartnern noch stärker engagieren.

? Was schätzen Sie an der Organisation der Pflege und den pflegerischen $A b$ läufen als gut gelungen ein?

Manske: Wir sind in einer ständigen Entwicklung. So haben wir beispielsweise die Pflegedokumentation komplett digitalisiert. Gute Entwicklungen weiter voranzutreiben, ist mein Ziel. Darum engagiere ich mich aktuell für das digitale Lernen in unserem Haus. Denn digitales Lernen ermöglicht es, aktuelle Themen schnell aufzugreifen und umzusetzen. So können zum Beispiel alle Mitarbeitenden direkt am Arbeitsplatz und bei laufenden Betrieb nicht nur theoretisch mehr über die Versorgung alter Menschen in einem Akutkrankenhaus lernen. Der Vorteil digitalen Lernens besteht darin, dass das Erlernte gleich praktisch umgesetzt werden kann. Die Lernenden erleben damit die Wirkung ihres Handelns und bekommen umgehend Rückmeldung.

? Was planen Sie, um gutes Personal zu halten und zu gewinnen?

Manske: Zur Gewinnung von Personal haben wir unser Bewerbungsmanagement verbessert. Inzwischen erhalten die Bewerber innerhalb eines Tages nach Einreichung der Unterlagen eine Rückmeldung und wir vereinbaren einen Gesprächstermin.

Um Mitarbeitende zu halten, ist auch der Fokus auf "Freizeit, Beruf und Familie" wichtig. Wir wollen deshalb mehr Arbeitsplätze schaffen, die diesen Bedürfnissen entgegen kommen. Da haben wir Nachholbedarf. Weil in der Pflege im Durchschnitt $80 \%$ Frauen arbeiten, sehen wir es als unsere Aufgabe an, in der Zeit von Mutterschutz und Elternzeit Kontakt zu innen zu halten. Wir müssen ihnen eine gute Wiedereinarbeitung ermöglichen und Arbeitszeitmodelle schaffen, die den persönlichen Bedürfnissen entsprechen, aber auch mit betrieblichen Belangen in Einklang zu bringen sind.

? Und welche Schwerpunkte setzen Sie sich selbst für 2017?

Manske: Wir haben es in der Pflege versäumt, den Part der Absolventen mit Bachelor- und Masterabschluss zu definieren. Was wir erreichen müssen, ist eine Funktionsdifferenzierung entsprechend der Qualifikation. Mir ist wichtig, dass akademische Ausbildung nicht "weg vom Bett" heißt. Gerade dort sollen die akade-

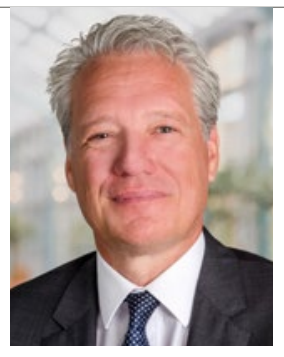

Rainer Manske Pflegedirektor im Unfallkrankenhaus Berlin

misch Qualifizierten wesentlich zum Theorie-Praxis-Transfer beitragen oder in spezialisierten Stationen verantwortungsvolle Aufgaben übernehmen. Dazu braucht es aber ein abgestuftes System für die Pflege. Es muss klar definiert werden, was ist Service, was sind pflegerische Hilfstätigkeiten und was ist Pflege auf einem hohen Niveau. Hier liegt die größte Herausforderung, diese ausdifferenzierten Funktionen angemessen in den Alltag eines Krankenhauses zu integrieren.

Das Interview führte Prof. Dr. Ingrid Kollak

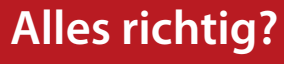

In diesem Monat erhalten Sie keine neuen Auflösungen.

Die korrekten Antworten der PflegeKollegs aus der September-Ausgabe können wir Ihnen erst im Februar verraten.

\section{Vorschau!} Die PflegeKollegs im Februar:

Ulcus Cruris versorgen

Ursachen - Wundauflagen - Druck ausüben

Hygiene

Desinfektion - Hauterkrankungen - Jeder Moment zählt 